\title{
Developing Biotherapeutics in the New Decade
}

Wilson S. Meng ${ }^{1,2} \cdot$ Erinc Sahin $^{3} \cdot$ Yi Wen ${ }^{4}$

Published online: 29 May 2020

(C) Springer Science+Business Media, LLC, part of Springer Nature 2020

In the last 5 years, the FDA has approved more than 40 therapeutic antibodies and antibody-drug conjugates. Despite the robust pipeline of the pharmaceutical industry, critical challenges remain to be addressed in bringing complex biologic therapies from discovery to approval. The steps toward transforming a novel immunoglobulin to therapeutic antibody involve a complex series of interdependent workflows woven throughout discovery, clinical and CMC development, and preparation of a strong regulatory filing. A successful program is ultimately crowned with commercialization and life cycle management of a drug product that meets the unmet medical needs of the patients who will benefit from its approval. In this thematic issue, we highlight to the communities of scientists, engineers, and clinicians regarding the current thinking and approaches used in advancing recombinant proteins through the drug discovery and development process. We selected original research articles and reviews on bioprocessing, developability and manufacturability of biologics, formulation and process development, and immunogenicity prediction of antibody and fusion protein-based biotherapeutics, in hopes of both updating the knowledge of those experienced in the field on recent developments, as well as providing some food for thought for those who are building the foundation of technical expertise in the early years of their careers.

The papers follow a thread in emphasizing that producing quality and patient-centric pharmaceutical products is a function of well-conceived active pharmaceutical ingredients

Wilson S. Meng

meng@duq.edu

1 Graduate School of Pharmaceutical Sciences, School of Pharmacy, Duquesne University, Pittsburgh, PA 15282, USA

2 McGowan Institute for Regenerative Medicine, University of Pittsburgh, Pittsburgh, PA 15219, USA

3 Global Product Development \& Supply, Bristol Myers Squibb, New Brunswick, NJ 08903, USA

4 Lilly Biotechnology Center, Lilly Research Laboratories, Eli Lilly and Company, San Diego, CA 92121, USA
(API), manufacturing processes, formulations, packaging, and devices. Suitable biological API should exhibit low immunogenicity risks determined using validated, highthroughput methods, as reviewed by Duke and MitraKaushik. Krishna reviewed the product-related factors, including aggregates, impurities, post-translation modifications, and excipients, that may drive immunogenicity. Highconcentration biologics drug products have been developed to extend dosing intervals, thereby improving patient compliance and pharmacokinetic profiles. But, the need for optimized formulation and container closure system to maintain the protein API in its native conformation during all stages of a product's lifetime cannot be overemphasized, as effectively summarized by Sahin and Deshmukh. The work by Mou and Jiang illustrated the technical advances in continuous crystallization of an excipient used in protein formulations. Thati and colleagues identified the critical parameters in the SCISSOR system that lay the methodological basis for the prediction of formulation risks upon administration. The ability to predict phase changes of API upon injection into tissues would be a decisive advantage in formulation development. Finally, to meet the demands of changing medical needs and market focus, the production yields of protein biologics must be improved. Schweickert and Cheng addressed this in their paper on the advances in genetic engineering of prokaryotic and eukaryotic bioproduction.

We predict that within this decade, end-to-end product optimization, in which cumulative changes in quality attributes are monitored and mapped by increased use of process analytical technologies, will become the standard strategy and expectation in biologics drug development. The efficiency of each approach will build upon the science and development of bioinformatics, protein engineering, novel analytical methods, optimal formulation and process, manufacturing, and data analytics.

Publisher's Note Springer Nature remains neutral with regard to jurisdictional claims in published maps and institutional affiliations. 